ON THE

\section{TREATMENT OF ULCER OF THE STOMACH.}

By BALTHAZAR W. FOSTER, M.D., M.R.C.P. LoND., PBOFESBOR OF CLINICAL MEDICINE IN QUEEN'S COLLEGE, PHYSICIAN TO THE QUEEN'S HOSPITAL AND THE GENERAL DISPENSARY, BIRMINGHAM.

THE potent influence of rest in the treatment of disease has only recently received that scientific consideration to which it is so specially entitled. Its efficacy as a therapeutic agent has been recognised mainly in the surgical domain of practice, or in the treatment of the diseased condition of parts endowed with functions which admit of a break in the continuity of their action without disturbance to the system at large. In Professor Hilton's eloquent Lectures, numerous convincing examples are advanced of the good results obtained by the application of mechanical and physiological rest to the treatment of many surgical maladies, and instances are not wanting to indicate its value in the management of cases more especially medical. The influence of this agent in the hands of the physician is, however, necessarily limited by the very nature of the functions of those viscera whose disorders he is called upon to treat. For in the present state of our knowledge the cessation of function in many of the internal organs means death, so intimately is the continuity of their action connected with the maintenance of life. The failing heart and the worn-out lungs can know no break in the life-long circle of their toil for as their continuous action means life, so their repose means death. Nevertheless, even in diseases connected with the functions of circulation and respiration, the influence of rest is not wholly excluded, but, on the contrary, forms in the present day an element of no small importance in our treatment. For although absolute rest, even for a short time, cannot be afforded to these organs, yet by diminishing the amount of their labour, and by removing the obstacles to normal action, we gain for them comparative, or, as it may be called, partial rest. In maladies of the nervous system, and in those of some of the excreting glands, no small part of our success would be lost were this weapon taken from our armoury; but it is more especially in diseases of the stomach, an organ for which we can obtain complete rest, that we find the greatest triumph is reserved for this therapeutic agent. This, to a certain extent, has been long recognised, and partial rest for disordered diges tion has formed the basis of treatment in the hands of all masters of our art.

The stomach, when seriously affected, teaches this lesson by its own acts, for in the pain caused by the ingesta, and in their rapid rejection, we have an unmistakable negative returned to the too officious efforts to restore health by the stimulus of food, when the soothing influence of rest is alone required. That the application of this remedy to the management of stomach diseases should have been early noticed is not surprising, considering that the indications are so evident, and the good results so rapidly follow its use. But it is surprising that rest should have been generally administered in serious organic diseases in such sparing quantities, such infinitesimal doses, at once too partial in its nature and too brief in its dura. tion. For some years past the value of a treatment which affords complete rest to the stomach in its serious disorders has been increasing in my estimation, and the application of the plan to the treatment of ulcer of the stomach and to many other lesions of the viscus has strongly confirmed my opinion. The nature of the digestive act is undoubtedly the great obstacle to repair in gastric ulcer. But nevertheless authors have contented themselves with enjoining only that partial rest for the stomach which a limited diet affords. There is abundant evidence that this mode of treatment is in many cases efficacious, and the case of Béclard may be well cited in its favour. That distinguished anatomist recognised clearly the necessity of rest, as complete as possible for the organ, and in treating himself for this affection, limited his diet to such a degree that it barely sufficed to support life. His almost total abstinence met its reward in the cure of his disease, and at his death the necropsy revealed the cicatrix of an old ulcer. By far the greater number of cases attending at our dispensaries and hos. pitals as out-patients are necessarily treated in this way, and thus week after week, and month after month, the wearing pain and the half starvation have to be endured, till a gradual recovery, too often with shattered health, is obtained, or, as often happens, the sudden gush of blood from an ulcerated vessel, or the rapid collapse of perforation, closes the tedious case.

On the other hand, with the in-patients in our charities, and with all who can obtain good nursing, we have, in the judicious combination of partial and complete rest, the best means of ensuring a speedy recovery. During the first stage of the treatment the patient should be kept strictly to the recumbent posture, all food or drink by the mouth entirely forbidden, and nutritive enemata frequently administered ; thus we procure for the stomach a long rest from all mechanical irritation, and from all physiological action. In the second stage, by a carefully regulated diet we should afford that partial rest necessary to perfect the cicatrisation, and to accustom the new tissue to the stimulus of the digestive act. The indications which we have to fulfil in the treatment of ulcer of the stomach are two-first, to promote the healing of the ulcer, and secondly, to alleviate the symptoms. By giving the viscus complete rest, from all physiological action, from all mechanical irritation, we remove the great impediments to the healing process, which consist in (1) the movements of the stomach consequent on the ingestion of food, and (2) in the mechanical and chemical irritation of the ulcerated surface produced by the food and gastric juice. The second indication is also fulfilled, for the pain and vomiting cease, and the hæmorrhage seldom if ever returns; while the patient, placed in the conditions for causing the least possible tissue-waste, is fairly nourished, and in many cases even strengthened, by the use of suitable nutritive enemata. In my hands this treatment has succeeded so well that I have every confidence in describing it as at once the most easy, the most rapid, and the most efficacious. In some twelve cases of gastric ulcer, and in several cases of ulceration from the action of corrosive fluids, as well as in other grave disorders of the digestive functions, have obtained from it the most satisfactory results. The two following cases will serve to illustrate my mode of treatment.

CASE 1.-J. H_- aged twenty, married, was admitted into the Queen's Hospital on June 20th, 1865.

History. - She has never been very strong, and has frequently suffered from attacks of indigestion. She was married at sixteen, has had one child, and has miscarried once. Before marriage she worked as a packer in a warehouse, and afterwards she was at a confectioner's, where the work was very heavy. Since her marriage she has not had much hard work, and has enjoyed improved health, especially whilst suckling. For the last twelve or thirteen months her health has been indifferent, and about four months back she was seized with severe pain in the epigastrium accompanied by headache and vomiting. For several months her food was frequently rejected, and about one month before her admission she vomited several ounces of blood, and passed about the same time several black, tar-like stools. The hæmatemesis recurred from time to time with the rejection of food; sometimes merely a streak or two, at other times several ounces of blood being expelled. The catamenia have recurred at the proper intervals during her illness, but she has had a leucorrhoeal discharge. Her bowels have lately been confined, and she has occasionally been annoyed by hæmorrhoids.

State on admission. - She wears an anxious expression ; face pale; lips anæmic. She complains of severe pain over the stomach, sometimes of a dull and burning character, at other times acute and lancinating. Appetite bad; and she fears to take food, as it intensifies the pain. The pain is referred to a spot immediately below the ensiform cartilage, where the tenderness on pressure is very marked, but limited to a spot about the size of half-a-crown. Shooting pains extend now and then from this centre all over the epigastric region, especially after food, when the pain is most severe, and is only relieved by vomiting. The tongue is moist, and covered with a whitish fur; urine healthy; liver and spleen normal in size. The examination of the chest detected no abnormality in the lungs; but an anæmic bruit was heard at the base of the heart, and along the great vessels. She was ordered to keep strictly to the recumbent posture; to have no food or drink by the mouth, except three ounces of milk mixed with half the quantity of lime-water, to be taken to moisten the mouth during the day; an enema of warm water to clear the lower bowels; and afterwards five nutritive enemata daily, of three ounces each, three to be composed of strong unsalted beef-tea, and two of milk and yelk of egg, with ten minims of tincture of opium in each. Ice to moisten the mouth.

On the following day (June 21st), the nurse having reported that the milk and lime-water were rejected as soon as taken, 
they were discontinued. The pain in the stomach a little better, as is also the headache. 'The tongue slightly furred.

June 23rd.-No sickness since all food by the mouth has been stopped. Tongue less furred; pain in the stomach less. The patient is not anxious, and admits that she feels better. The enamata have been retained very fairly, only one having been returned.

24th. - The patient feels better, but very weak; the pain nearly gone; the tenderness on pressure much diminished. She has a headache, and feels very thirsty. The tongue red and rather dry. "The rectum slightly irritahle, a portion of the enemata having returned with some freces three times on the day before. Pulse 84 .

26th.--Complains of headache and slight nausea. Pain in the stomach much less. Pulse 60 ; bowels irritable. Ordered twenty minims of tincture of opium, with three of the enemata. 29 th. - Has improved daily since the last note. The irritability of the rectum has been checked by the tincture of opium. She has felt no nausea or pain; pressure over the stomach now elicits scarcely any sign of tenderness; headache much better; tongue red, dry, and furred at the edges; says she feels very weak, and desires to eat. Ordered milk and lime-water, of each half an ounce, every two hours, and only three injections daily.

30th.-Much better; tongue clean. The milk has caused neither pain nor sickness. Ordered milk thickened with a little corn-flour, and to discontinue the enemata after July lst, and then to take one ounce of fried sole for dinner. Pulse 84, feeble.

July 4th.-Much improved; tongue clean and moist; pulse 80 , stronger. She has taken milk with corn-flour and arrowroot, also the fish, without any pain or uneasiness in the stomach. Ordered a small piece of chicken for dinner.

5th.- To take the following twice daily:-Iron and citrate of quinia, four grains; infusion of calumba, one ounce.

10th. -The patient is up, and walking about the ward; feels no pain after eating; enjoys her food, and has a good appetite; tongue clean; pulse 80 ; no tenderness on pressure over the stomach. At this time she was fit for discharge as far as the gastric symptoms were concerned; but she was kept in the hospital a fortnight longer on account of the anæmia, which gradually disappeared, and with it the murmur at the base of the heart.

She showed herself once a month for several months after her discharge, and on all occasions she was healthy and free from all gastric symptoms.

CASE 2.—J. J—, aged twenty-two, dressmaker, was admitted on March 4th, 1866, into the Queen's Hospital.

History. - About one month before her admission she applied as an out-patient, stating that she suffered very much from indigestion and a severe pain in the stomach, increased by taking food; she had occasionally vomited blood with her food. These symptoms began, she said, about eighteen months previously, and increased in intensity up to the time of her appearance at the hospital. Before this illness she had always had good health. She was ordered some powders containing bismuth and powdered opium, and had full directions given her concerning her diet. She was also told to return immediately to the hospital if. the vomiting of blood recurred. The hæmatemesis having again appeared on March 20th after a rather full meal, she was admitted on March 22nd.

March 23rd.-Her face is pale and slightly puffy; tongue Habby and slightly furred. She complains of slight headache, and of pain in the stomach and constant sickness. The pain comes on chiefly after eating; it shoots over the stomach, and is also felt very badly in one spot posteriorly in the left vertebral groove. She is afraid to eat on account of vomiting. The stomach will not even retain milk in very minute quantities. On examination, the pain is found to be situated just below the ensiform cartilage, and to the left side of the middle line. The area of tenderness on pressure is about the size of a halfcrown. Pulse 70, weak and small. Heart-sounds normal. Lungs healthy. Liver and splenic dulness natural. Urinary organs healthy. Temperature normal. Ordered to take no food or drink by the mouth; to have, every four hours, an enema composed alternately of milk with yelk of egg, and strong unsalted beef-tea, with fifteen minims of tincture of opium added to each; to be kept strictly to the recumbent posture; and to have her mouth frequently moistened with water by the nurse.

25th. - The pain in the stomach is still felt, but not so badly. The sickness has altogether ceased. Tongue cleaner and drier; pulse 80 . The treatment has been strictly followed, and the enemata have been retained. 26th. - No sickness or feeling of nausea; the pain much better, but the epigastric region as defined above still tender on pressure. Pain in the back better. She feels low, and very hungry. Tongue dry and slightly furred. Her bowels were moved twice yesterday, but not till two hours after enemata. Pulse 68, weak and compressible. Injections to be continued, with ten grains of pepsine added to each.

28th. - Much easier in the stomach; tenderness less. The pepsine has, however, irritated the bowel, and caused the speedy rejection of the enemata. Slight pain has also been felt along the sigmoid flexure. The pepsine ordered to be omitted, and enemata of milk and egg, with twenty-five minims of tincture of opium in each, to be given. Pulse 58, weak.

29th. - She feels much better. No pain in the stomach or back, and scarcely any tenderness on pressure. Tongue furred and dry; pulse 68 , stronger. The enemata have been well retained, and the tenderness in the course of the lower bowel has diminished. Ordered half an ounce of milk every two hours, and two injections daily.

31st. - The milk has been retained, and caused no pain or uneasiness. Tongue moist, and less furred. The milk to be thickened with corn-flour.

April 1st. - The milk has caused no pain or sickness. Tongue clean. Scarcely any pain on pressure over the stomach. The milk and corn-flour to be continued.

2nd.-Tongue slightly furred, but she has had no pain in her stomach, and has enjoyed her food. The enemata, having irritated the bowels slightly, were ordered to be discontinued, and warm ponltices to be applied over the lower part of the abdomen. One ounce of uncooked mutton, chopped very fine, to be given in three portions during the day; also two ounces of sherry.

3rd.-Very much improved. The meat has agreed very well. Ordered to continue it in gradually increasing quan tities.

5th.-Much improved; tongue clean ; no pain or sickness after food; no pain on pressure; appetite good. The patient expresses herself as almost well. Ordered a little of the breast of a chicken, with bread and two ounces of sherry.

10th.--Discharged as quite well. She was directed to be very careful in her diet for a week or so, and to return most gradually to her ordinary food. She came to the hospital several times after her discharge, and was quite free from all her former dyspeptic symptoms, and had returned with pleasure to her ordinary habits of life.

The foregoing notes contain a fair description of the management of all the cases of ulcer of the stomach which I have treated for some three years past in the Queen's Hospital.

As I have previously described it at length, ${ }^{*}$ I need not dilate now on the treatment, which resolves itself, in all cases, into two periods, the first of which we may call the stage of absolute or complete rest, the second that of partial rest. The first may last from three to ten days, or even longer ; and during this time the nutritive enemata must be solely relied upon for the support of the patient. No food and no fluid must enter the stomach. In the second stage, the nutritive enemata may be continued for a day or two, while the most scrupulous care is exercised in the choice of food, and the most rigorous supervision given to its administration. The addition of the tincture of opium to the injections from the very beginning is advantageous, not only on account of the beneficial action of the drug on the system, but more especially on account of its soothing effect on the rectum. Irritation of the bowel is generally prevented by its use, and when it does occur, is nearly always checked by an increased dose. This is a fact of great importance where the nourishment of the patient wholly depends on the adaptibility of the bowel to its novel office.

The pepsine added to the injection in one of the cases caused, as is reported, considerable irritation of the bowel; and I have since found the same effects following its use. The addition was made as an attempt to apply a suggestion which appeared in a very able reviewt of a former paper of mine on this subject. In the article referred to, Dr. P. Sonsino proposed the use of enemata composed of artificially digested food. The use of the pepsine was a rough attempt to utilise his thought Its ill success, however, by no means diminishes the value of the suggestion; and I hope soon to report the success of a

Vide Dublin Quarterly Journal, May, 1864: "Clinical Observations on Diseases of the Stomach" (Cornish Bros., Birmingham, 1864.)

Lecture on the Treatment of Gastric Uleer (Brit. Mied. Jour., June 3rd, 835 )

+ Vide L'Imparziale, Nor. 16th, 1865. 
more elaborate attempt to carry ont Dr. Sonsino's valuable proposal. The researches of $\mathrm{Mr}$. Savory ${ }^{*}$ in this country, and the more recent experiments of $M$. Demarquayt in France, toach as how great is the absorptive power of the rectum. In all cases in which the stomach refuses to act, or in which rest is likely to benefit the viscus, it is, I submit, our duty to ntilise this power. We find this mode of treatment a powerful aid in combating many morbid conditions other than ulcer of the stomach; and, in illustration, I may specially mention the uncontrollable vomiting which so often accompanies pregnancy.

My friend Mr. Baines, of this town, has recently sent me the notes of two cases in which he adopted the treatment by complete rest with the best results after all the usual remedies had failed. In one case the patient" was fed in this manner for more than four weeks. In many cases, now unfortunately matters of history, the same judicious management would, I believe, have proved equally efficacious. It has been often remarked to me, that the treatment of uleer of the stomach and other conditions by this plan, which I have recommended, can never become general on account of its indelicacy. For my own part, I have always found patients suffering from ulcer of the stomach too happy to find any means of escaping from the agonising pain to think for a moment of such an objection, and, when hæmorrhage in any quantity has once occurred, too eager to grasp at any hope of cure. False delicacy soon kicks the beam when the love of life is placed in the opposite scale. Birmingham, April, 1808.

ON

\section{CHANCRES IN ADULTS AND CHILDREN, ATTACKING UNUSUAL POSITIONS, ASSO- CIATED WITH SYPHILIS.}

\author{
BY THOMAS BRYANT, F.R.C.S.,
} ASSTSTANT-SUBGEON TO GEY'S HOSPITAL.

The records of cases which differ from the ordinary run of recognised disease are always of interest, and for diagnostic purposes cannot be too closely studied. In syphilitic affections it cannot be too well known that chancres of every form may be found in parts far distant from the genital organs, and that they may be produced in very different ways from the common kind of venereal affections. In a diagnostic point of view, it is a matter of considerable importance that these facts should be recognised; and as a contribution to the clinical aspect of the question, the following cases may not be without interest.

The cases are arranged in order. The first are those of chancres as attacking adults; the last as affecting children.

CASE 1. Chancre of the upper lip, with constitutional syphilis. Annie T-, aged twenty-two, a single woman, came under my care at Guy's Hospital on Sept. 13th, 1858, with a chancre on her upper lip of three months' standing. It was the size of a sixpence, and very indurated. For two months she had suffered from sore-throat, and had the staining of the skin which follows a specific eruption. A gland beneath the jaw was also indurated. Under the influence of iodide of potassium she rapidly convalesced.

CASE 2. Chancre of the upper lip and specific lichen.-Amelia $\mathrm{W}-$, aged twenty-one, a single woman, came under my care at Guy's Hospital in May, 1860, with a chancre on her upper lip of six weeks' duration. It bad commenced as a fissure, and gradually spread. When seen it was as large as a sixpence. For two weeks she had also suffered from a papular eruption all over her body (specific lichen). Some Plummer's pill was given at night, and tonic mixture during the day, and in six weeks she was discharged cured.

CASE 3. Chancre of the lower lip, sore-throat, maculce, and indurated glands. - John $\mathrm{H}-$, aged thirty, came under my care at Guy's Hospital, April 29th, 1861, with a chancre on his lower lip of one month's duration, sore-throat, and indurated submaxillary glands. Some specific maculæ also appeared over his body when under treatment. He rapidly convalesced under the influence of the iodide of potassium.

CAse 4. Chancre on lower lip; specific maculoe and indu-

$*$
+ rated glands. - Emma F_, aged twenty-two, came under my care in January, 1862, with a chancre on her lower lip, maculæ over the face and body, and indurated submaxillary glands. The chancre had existed eight weeks; the other symptoms about six. A grain of iodide of mercury was given every night, and three grains of iodide of potassium with cinchona three times a day. In about two months she was convalescent.

CASE 5. Chancre at the corner of the mouth; no constitutional symptoms; inflamed submaxillary glands. - Ann I__ aged seventeen, came under my care at Guy's Hospital on the lith of July, 1864, with a soft chancre at the corner of her month, of three weeks' duration, and inflamed submaxillary glands. Under tonic treatment the sore healed and the inflamed glands subsided, the patient leaving the hospital cured in about one month.

In none of the eases just related could any history be obtained as to the means by which the lips became inoculated with the disease; and in none of the examples in which the chancre occurred in women was there any evidence of any vaginal affection. In the single male example the penis was clean.

CASE 6. Chancre on the forearm; constitutional syphilis.-A man came to me in November, 1866, with an indurated chancre on his left forearm, the size of a shilling, of six weeks' standing. When under observation a specific lichen came out all over his face and body. He had no disease on his penis, nor was he able to account for the chancre on his forearm. By taking quinine with the iodide of potassium all his symptoms disappeared.

CASE 7. Chancre on the nipple; constitutional symptoms. -In 1865 a girl aged nineteen came to me with an undonbted chancre of the nipple, which had existed a month; in another month specific maculæ appeared over the body, with sore throat. She had no vaginal disease of any kind; but she admitted that her "young man," who had an eruption on his face and hands, had manipulated her breast and had kissed it. Under treatment with iodide of potassium and bark she convalesced.

CASE 8. Chancre on the fingers; specific lichen; recovery.Nathaniel S_- aged twenty-five, a labourer, came under my care, at Guy's Hospital, on Sept. 15th, 1864, with three chancres on the tips of three fingers of the right hand of three months' duration. He had also a specific lichen all over his body of six weeks' standing. His penis was free from disease. He had grazed his fingers at his work a few days before the sores appeared, and had "fingered women." By the use of iodide of potassium and bark he rapidly recovered.

CASE 9. Chancre on the cheek, specific maculae, and sorethroat, following a scratch from a man who was suffering from syphilis. - William H-, aged twenty-two, came to me, at Guy's Hospital, on Nov. 17th, 1866, with a large chancre on his left cheek, with an indurated base of nine weeks' duration. It appeared after a scratch he had received from a fellowlabourer who had syphilis. The cervical glands in the left side were much indurated. On the 15th of December maculæ appeared over his face and body, and sore-throat soon manifested itself. With quinine and iodide of potassium all the symptoms subsided. Un the 4 th of February the chancre had healed; and by the 18th of March every other symptom had disappeared.

CAsE 10. Chancre of the lip in a boy; constitutional syphilis.Thomas $J-$, aged seven years, a healthy lad, was brought to me, at Guy's Hospital, on Feb. 14th, 1867, with a chancre on his lower lip of three weeks' duration. He was presented by his father, who had been under my care some years previously with a tubercular syphilitic disease of the face and body after a chancre which he had contracted some months before. Three rears ago, also, he had again been under my care for a syphilitic cellular membranous ulcer on the leg. When he came with his son his tongue was slightly affected with disease. Specific tubercles and scales subsequently appeared over the boy's face and body. Under the iodide of potassium the boy is now well. The father stated that he was very fond of his boy, and was constantly kissing him. It is probable that it was by such means that the disease had been conveyed.

CAsEs 11, 12. Syphilis in two children of the same family; fother and mother healthy.-David $\mathrm{G}-$, aged seven, came under my care on March 15th, 1866, with a specific tubercular eruption about the scrotum and thighs, also about the neck. It 